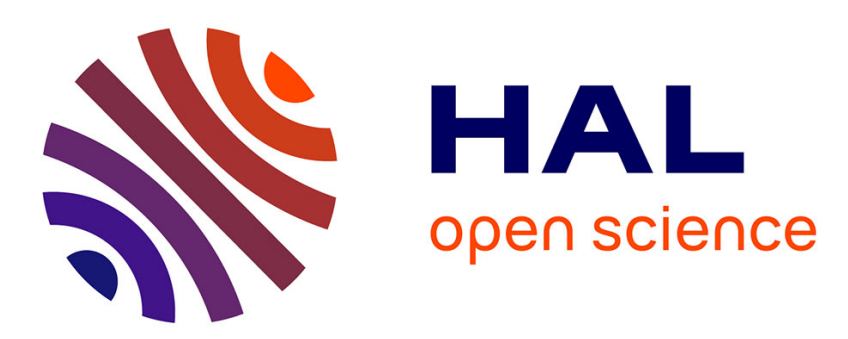

\title{
A Post-Processing Integral Formulation for the Computation of Magnetic Field in Conductors
}

\author{
Arnaud Guibert, Jean-Louis Coulomb, Olivier Chadebec, Corinne Rannou
}

\section{To cite this version:}

Arnaud Guibert, Jean-Louis Coulomb, Olivier Chadebec, Corinne Rannou. A Post-Processing Integral Formulation for the Computation of Magnetic Field in Conductors. IEEE Transactions on Magnetics, 2011, 47 (5), pp.1450 - 1453. 10.1109/TMAG.2010.2102341 . hal-00595014

\section{HAL Id: hal-00595014 https://hal.science/hal-00595014}

Submitted on 23 May 2011

HAL is a multi-disciplinary open access archive for the deposit and dissemination of scientific research documents, whether they are published or not. The documents may come from teaching and research institutions in France or abroad, or from public or private research centers.
L'archive ouverte pluridisciplinaire HAL, est destinée au dépôt et à la diffusion de documents scientifiques de niveau recherche, publiés ou non, émanant des établissements d'enseignement et de recherche français ou étrangers, des laboratoires publics ou privés. 


\title{
A Post-Processing Integral Formulation for the Computation of Magnetic Field in Conductors
}

\author{
A. Guibert ${ }^{1}$, J.-L. Coulomb ${ }^{1}$, O. Chadebec ${ }^{1}$, and C. Rannou ${ }^{2}$ \\ ${ }^{1}$ Grenoble Electrical Engineering Lab - Université de Grenoble (Grenoble-INP/UJF/CNRS), BP 46, 38402 Grenoble, France \\ ${ }^{2}$ BCRM de Brest, GESMA - Groupe d'Etudes Sous-Marines de l'Atlantique CC 42 - 29240 Brest Cedex 9, France
}

\begin{abstract}
We present here an original method of computing the magnetic induction in a conductor thanks to a post-processing from the boundary elements method (BEM). The paper gives the main keys of the algorithm, from the theoretical aspect as well as the practical one. This general formulation is then applied to simulated cases and finally to a real underwater electric system under cathodic protection.
\end{abstract}

Index Terms-Computation theory, conductors, magnetic fields.

\section{INTRODUCTION}

$\mathbf{C}$ OMPUTING the induction flux in a conductive media seems to be mastered since the appearance of the finite element method (FEM). Indeed this method consists in meshing the all structure studied in tetrahedral elements, which allows the computation of any electromagnetic values from the knowledge of a physical behavior and a part of boundary values. But this method has two main drawbacks.

- When computing values (such as the magnetic induction) in the conductive meshed media (containing currents) with volume integration, obvious singularities appear, while dividing by distances close to zero. Solutions commonly used are to simply take this element out of the calculation, which is physically wrong.

- For infinite (or semi-infinite) conductive medias (also called external problems), it is obviously impossible to mesh a sufficient volume. In this case, an Infinite Box technique is commonly used, with a precision depending on its dimension and the number of meshing elements. This box size has to be tested to find the best compromise.

This method is efficient in internal cases (when currents are "contained" in a finite conductive media) where no currents spread at the computation point. As a main drawback, compromise between meshing precision (and hence results accuracy) and memory available has to be well chosen. But the advantage of working with sparse matrixes, permitting the use of an important quantity of elements, is undeniable.

Since the recent developments of computational capabilities, huge mathematical calculations are available. This has led to an interest renewal for the boundary elements (BEM), working with fulfilled matrixes. Indeed, this last limit numerically restricts the number of admissible meshing elements. But contrary to the FEM, it works with surface elements, avoiding trouble for magnetic computations in the conductive media. Moreover, this method can be used in external cases, representing the infinity bound by a far equipotential limit where no currents spread away. The reader can find more precisions on external cases in a previous publication [1]. The following part first deals with the calculation formulation.

Manuscript received May 30, 2010; revised November 22, 2010; accepted December 11, 2010. Date of current version April 22, 2011. Corresponding author: A. Guibert (e-mail: arnaud.guibert@g2elab.grenoble-inp.fr).

Color versions of one or more of the figures in this paper are available online at http://ieeexplore.ieee.org.

Digital Object Identifier 10.1109/TMAG.2010.2102341

\section{General Formulation of the COMPUTATION Method}

Let us consider a conductive, linear, homogeneous and isotropic domain $\Omega$ in which flows a $\mathbf{j}$ static current density. Laplace equation is verified. The classical equation to compute the magnetic flux density is the Biot-Savart law:

$$
\mathbf{B}(P)=\frac{\mu}{4 \pi} \cdot \iiint_{\Omega} \mathbf{j}^{\wedge} \frac{\mathbf{r}}{r^{3}} d \Omega
$$

In this last equation, the magnetic flux density is computed at a $P$ point from the knowledge of currents repartition $\mathbf{j}$ in $\Omega$ with $\mu$ permeability. Remembering that $\mathbf{B}$ computations will further be suited in conductors, this last equation induces singularities. Moreover, for infinite cases (structures immerged in infinite water), volume meshing is hard to apply.

\section{A. Transformation of This Integral}

A main property of the Gradient and its application to the previous equation give the following equations:

$$
\begin{aligned}
\mathbf{u}_{\mathbf{r}_{j}} \frac{1}{r_{j}^{2}} & =-\operatorname{grad}\left(\frac{1}{r}\right) \\
\mathbf{B}(P) & =-\frac{\mu}{4 \pi} \cdot \iiint_{\Omega} \mathbf{j}^{\wedge} \operatorname{grad}\left(\frac{1}{r}\right) d \Omega \\
& =\frac{\mu}{4 \pi} \cdot \iiint_{\Omega} \sigma \cdot \mathbf{E}^{\wedge} \operatorname{grad}\left(\frac{1}{r}\right) d \Omega .
\end{aligned}
$$

Another property of the gradient (where $\mathbf{a}$ is a scalar and $\mathbf{b} \mathbf{a}$ vector) allows a new transformation of the formula:

$$
\begin{aligned}
\mathbf{a}^{\wedge} \operatorname{grad}(b) & =b \cdot \operatorname{rot}(\mathbf{a})-\operatorname{rot}(b \cdot \mathbf{a}) \\
\mathbf{B}(P) & =-\frac{\mu \cdot \sigma}{4 \pi} \cdot\left(\iiint_{\Omega} \frac{1}{r} \cdot \operatorname{rot}(\mathbf{E}) d \Omega\right. \\
& \left.\quad-\iiint_{\Omega} \operatorname{rot}\left(\frac{\mathbf{E}}{r}\right) d \Omega\right) .
\end{aligned}
$$

As we are in a static case, the following equality is verified:

$$
\operatorname{rot}(\mathbf{E})=-\frac{\partial \mathbf{B}}{\partial t}=\mathbf{0}
$$


Finally, the new equation obtained is

$$
\mathbf{B}(P)=\frac{\mu \cdot \sigma}{4 \pi} \cdot \iiint_{\Omega} \operatorname{rot}\left(\frac{\mathbf{E}}{r}\right) d \Omega .
$$

\section{B. From a Volume to a Surface Integral}

Ostogradsky formulas allow projecting a volume integral on its surfaces. For $\mathbf{a} \mathbf{b}$ vector and $\mathbf{n}_{\mathbf{e x t}}$ the normal vector of the surface, we have the following transformation:

$$
\iiint_{\Omega} \operatorname{rot}(\mathbf{b}) d \Omega=\iint_{S(\Omega)} \mathbf{n}_{\mathrm{ext}}^{\wedge} \mathbf{b} \cdot d S(\Omega) .
$$

This lead to a new formula for the induction computation:

$$
\begin{aligned}
\mathbf{B}(P) & =\frac{\mu \cdot \sigma}{4 \pi} \cdot \iint_{S(\Omega)} \mathbf{n}_{\mathrm{ext}}^{\wedge} \frac{\mathbf{E}}{r} \cdot d S(\Omega) \\
& =\frac{\mu \cdot \sigma}{4 \pi} \cdot \iint_{\mathrm{S}(\Omega)} \frac{\mathrm{E}_{\tau}}{\mathrm{r}} \cdot \mathrm{u}_{\text {vect }} \cdot \mathrm{d} \mathrm{S}(\Omega) .
\end{aligned}
$$

Here, $\sigma$ is the domain conductivity, $\mathrm{E}_{\tau}$ is the tangential electric field. $\mathbf{u}_{\mathbf{v e c t}}$ is the unitary directional vector of the vector product between the electric field $\mathbf{E}$ and the surface normal vector. An analytic computation is too complicated to realize with complex boundaries, so a numerical approach is followed with a surface meshing in $N$ elements. A zero order approximation (quantities set constant by meshing elements) is admitted with a point matching approach (computations at the geometric center of elements). This approximation gives sufficient precision for our study and leads to

$$
\mathbf{B}(P)=\frac{\mu \cdot \sigma}{4 \pi} \cdot \sum_{i=1}^{N} E_{\tau_{i}} \cdot \mathbf{u}_{\mathrm{vect}, i} \cdot \int_{S_{i}(\Omega)} \int_{r_{i}} \frac{1}{r_{i}} \cdot d S_{i}(\Omega) .
$$

In this new expression, the only unknown is the electric field $\mathbf{E}$ at the geometric center of each surface element. This computation is possible with the BEM [1] and leads to get $\mathbf{u}_{\text {vect }}$ and $\mathrm{E}_{\tau}$

\section{Computing E on the Boundaries With the BEM}

As said before, the main problem remaining is the computation of the electric field on the boundaries. The numeric formula to compute it if using BEM is directly shown here [1]:

$$
\begin{aligned}
\mathbf{E}= & \frac{1}{2 \pi} \cdot \sum_{j=1}^{N} \varphi_{j}\left(Q_{j}\right) \cdot \iint_{S_{j}(\Omega)}\left(-3 \cdot \frac{\left(\mathbf{r}_{\mathbf{i j}} \cdot \mathbf{n}\right) \cdot \mathbf{r}_{\mathbf{i j}}}{r_{i j}^{5}}+\frac{\mathbf{n}}{r_{i j}^{3}}\right) \\
& . d S_{j}(\Omega)-\frac{1}{4 \pi} \cdot \sum_{j=1}^{N} \frac{\partial \varphi_{j}\left(Q_{j}\right)}{\partial n_{j}} \cdot \int_{S_{j}(\Omega)} \int_{-}-\frac{\mathbf{r}_{\mathbf{i j}}}{r_{i j}^{3}} \cdot d S_{j}(\Omega) .
\end{aligned}
$$

The $\varphi$ and $\partial \varphi / \partial \mathrm{n}$ at the $j$ geometric centers are known thanks to a previous BEM at the end of the resolution step. Only $2 \mathrm{~N}$ integral terms remain to be calculated:

$$
\mathbf{H}_{\mathbf{i} \mathbf{j}}^{\prime}=\iint_{S_{j}(\Omega)}\left(-3 \cdot \frac{\left(\mathbf{r}_{\mathbf{i j}} \cdot \mathbf{n}\right) \cdot \mathbf{r}_{\mathbf{i j}}}{r_{i j}^{5}}+\frac{\mathbf{n}}{r_{i j}^{3}}\right) \cdot d S_{j}(\Omega)
$$

$$
\mathbf{T}_{\mathbf{i j}}^{\prime}=\iint_{S_{j}(\Omega)}-\frac{\mathbf{r}_{\mathbf{i j}}}{r_{i j}^{3}} \cdot d S_{j}(\Omega) .
$$

Those two quantities can be easily computed (by a numerical approach with Gauss points), except when $i=j$. In this case, $r$ is null and the computation diverges.

We first focus on the computation of $\mathrm{H}_{\mathrm{ii}}^{\prime}$, composed of two components: as we integer an element on itself, the $\mathbf{r}$ vector will always be contained in the surface elements, and so perpendicular to the normal vector $\mathbf{n}$. Their scalar product is null and so is the first component. The second term is oriented in the normal direction (as $\mathbf{n}$ is the only vector appearing). Its tangential component will obviously be null too. Finally, the singularity apparently introduced by the $\mathrm{H}_{\mathrm{ii}}^{\prime}$ term is removed.

The case of the $\mathrm{T}_{\mathrm{ii}}^{\prime}$ computation leads to more difficulties. Indeed, no simplification is possible in this integral term. Fortunately, some references give clues on induction computation from polyhedral sources by a projection of volume integral to a surface one [2]. The calculation point is no more in sources, avoiding the singularity, and the integral term can be computed. An example in magnetostatic can be found in [3].

After obtaining those problematic integral terms, the tangential field at all geometric centers is available, and so is the induction field anywhere in the conductor.

An improvement stands in locating neighbors of a $P$ element to compute their strongest influence (on $P$ ) more precisely. As the general computation is numerically made with Gauss point, this means adding more integration points in those neighbor elements.

The developed method is applicable to any conductive media but let us focus on a precise scope.

\section{Using The Method IN CORROSION Simulation}

The aim of this part is to predict the magnetic induction due to electrochemical phenomenons: protection against corrosion, also called cathodic protection.

\section{A. Cathodic Protection Context}

During its operating life, a steel underwater structure has to face to corrosion, which damages it physically. To fight against this phenomenon, two main solutions exist.

- The Sacrificial Anode Cathodic Protection (SACP), which places a metal (less noble than the iron contained in steel) anode on the surface to protect. This new anode will be corroded instead of the structure.

- The Impressed Current Cathodic Protection (ICCP), which role is to inject currents in the water through platinum anodes. This places the steel in its passivation area and protects it.

In both cases, cathodic protection leads to a circulation of currents in a conductive media (which is the salted seawater) from the anode to the cathode [4].

\section{B. Case Studied and Primary Results}

The first structure studied is a parallelepipedic steel mock-up immerged at the centered bottom of a finite salted water volume $\left(\sigma=5 \mathrm{~S} . \mathrm{m}^{-1}\right)$. This steel mock-up is covered with an isolating painting with two rectangular defects $(0.08 \mathrm{~m} \times 0.02 \mathrm{~m})$. To protect it, an ICCP system is put at the middle of the structure with a square platinum anode $(0.02 \mathrm{~m} \times 0.02 \mathrm{~m})$. The meshed structure is shown in Fig. 1. 

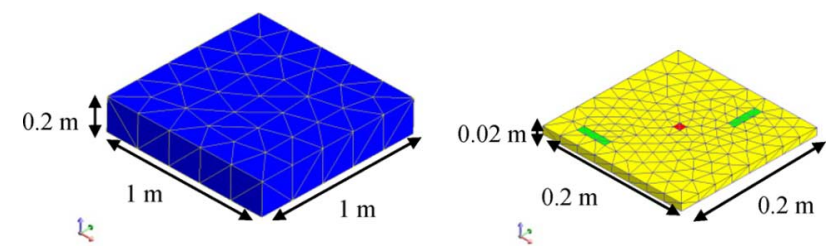

Fig. 1. Meshing of the seawater volume (on left); the steel mock-up (on right), with a 1 red anode, 2 green defects, 1 yellow isolated part; units in meters.
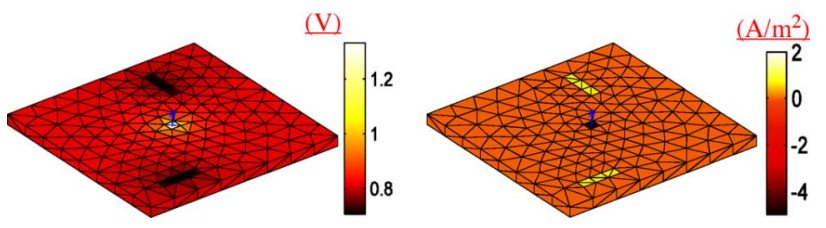

Fig. 2. BEM results on the mock-up, with potentials (left) in $\mathrm{V}$ and current densities (right) in $\mathrm{A} / \mathrm{m}^{2}$.

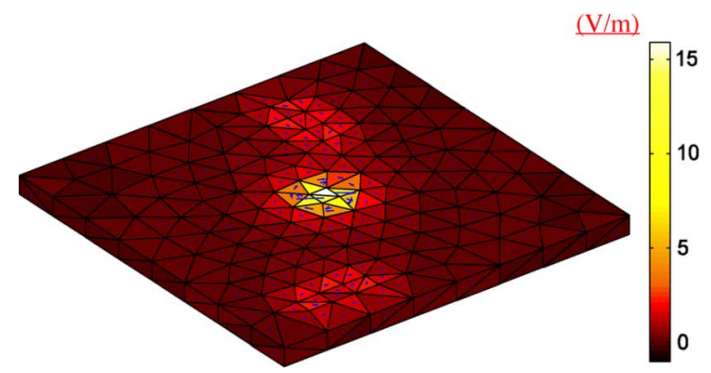

Fig. 3. Tangential electric field on the mock-up with its modulus in V/m.

Once this meshing is done, the BEM can be applied to get the missing boundary conditions $\varphi$ and $\partial \varphi / \partial \mathrm{n}[5]$. As we are in an ICCP case, the anode $\partial \varphi / \partial \mathrm{n}$ (equal to a $5 \mathrm{~A} / \mathrm{m}^{2}$ current density within the conductivity $\sigma$ ), the cathode polarization law (linking $\partial \varphi / \partial \mathrm{n}$ and $\varphi$, nonlinear) and the isolated part $\partial \varphi / \partial \mathrm{n}$ (equal to zero) are known. The nonlinear BEM (with a Newton Raphson algorithm, for example) leads to the boundary conditions shown in Fig. 2.

As those results are obtained, a post-processing step allows obtaining the magnetic induction.

\section{Magnetic Induction Results}

As said in the second part of the paper, the induction computation needs the knowledge of the tangent electric field on the boundaries. A first use of formula (9), with a singularities pretreatment and neighborhood detection, gives the total electric field. It is then possible to extract its tangential component (Fig. 3).

This extracted component rotates around the current sources, respecting the Gauss law. The $\mathrm{N}$ integral terms of (8) are then calculated to finally obtain the magnetic induction $\mathbf{B}$, here computed on a grid in the water, $0.02 \mathrm{~m}$ above the mock-up $\left(\mu=\mu_{0}\right)$ (Fig. 4).

To compare these results, a simulation with a FEM software (Flux3D of the CEDRAT society) is realized. This comparison is possible thanks to FEM adjustments: first an optimal meshing of the structure is empirically made. Then the Biot-Savart equation is computed in the whole domain from the knowledge of the sources repartition in the conductor (electric field on the boundaries is not needed). Finally a post-processing step of re-evaluation of the singularities is performed. The results on the same grid are shown in Fig. 5.

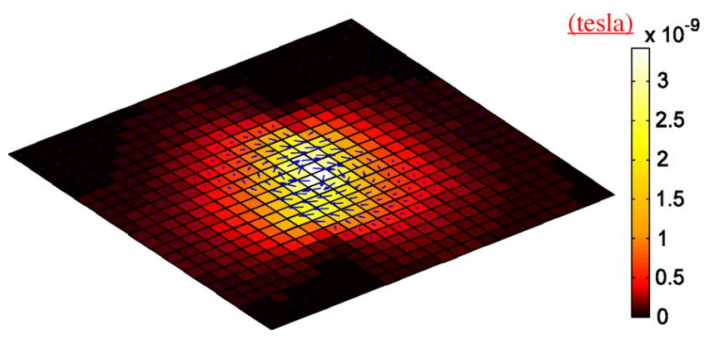

Fig. 4. Magnetic induction results on the grid with direction vectors and norm isovalues, color scale in tesla.

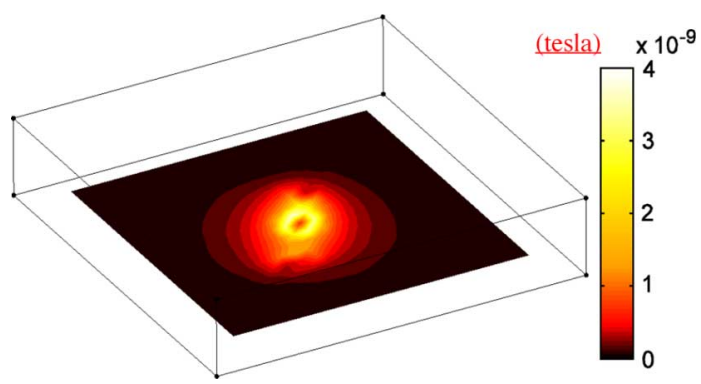

Fig. 5. Magnetic induction on the grid with norm isovalues, color scale in tesla.

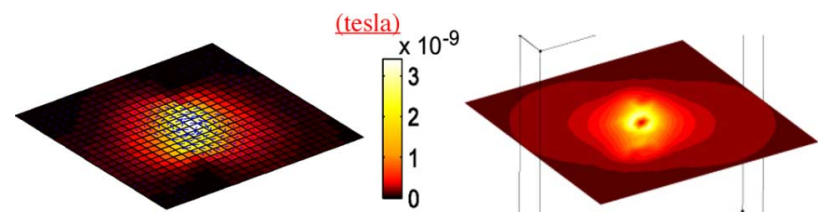

Fig. 6. Induction results on the grid with norm isovalues, color scale in tesla, from BEM (on left) and FEM (on right, with Fig. 5 color scale).

Results obtained are roughly the same (even when meshing more the structure) with less than $5 \%$ of the root square difference between the two results. As said before, the main drawbacks of the FEM are computations at sources location and choosing a compromise between refinement of the meshing (solution accuracy) and memory available. This does not happen with our method but the mandatory post-processing step to get electromagnetic values in the domain can be considered as a restraint.

\section{Quick Results in External Problem}

A similar type of computation and comparison can be made in external problems. Indeed, the appearance of an infinite bound only induces a few changes in the computation (which are not noted here for concision reasons). The comparison of the results is shown in Fig. 6.

The results (less than $5 \%$ difference) and conclusions are similar to the previous part. The Infinite Box introduces a new constraint with a compromise to choose between precision and meshing density. Our method is more efficient in this case.

\section{Application in a ReAl CASE}

One primary goal of world's Marines is to predict vessels' electromagnetic field, particularly to evaluate mines threat (more sensitive to magnetic field than electric one) during operations. Different components of this field are known, such as hull magnetization, motors' fields or corrosion currents. As the first two can now be easily predicted, Corrosion Related Magnetic field (CRM) has become one main difficulty. This is especially due to the presence of sources (corrosion currents) 


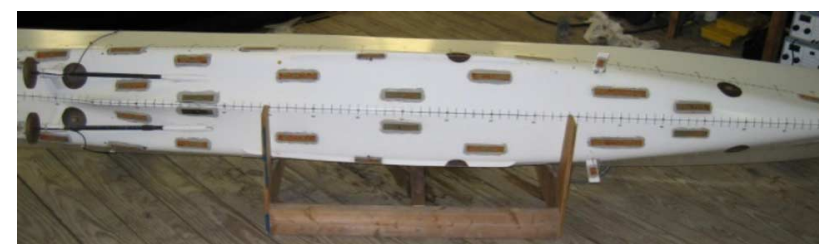

Fig. 7. 1:40th ship mock-up (underside) with rectangular cathodes and anodes in round dielectric patches.

where the field is searched. As said before, the developed method overcome this difficulty. Moreover, for a so complex hull structure, main FEM difficulties stand in the volume meshing of the mock-up with an Infinite Box. Our method only needs a surface meshing and brings more simplicity in this case.

An algorithm of corrosion diagnosis has been presented in a previous communication [1]: from a set of close electrical measurements in the water, main goal is to obtain boundary conditions on the structure surface (like in Fig. 2), and thus corroded areas.

An interesting use of our method would be, from a set of close electrical measurements, to find corroded areas on the hull and then predict the magnetic field at a deeper depth. This paragraph considers a complex geometry, representative of a ship hull under ICCP. Some electromagnetic measurements are realized below this mock-up, at 2 different depths. The closest one helps finding corroded areas and the furthest is used to predict the magnetic field. Unfortunately, as experimentations were made to check electric field prediction tools, magnetic measurement cannot be used as direct validation.

A frigate mock-up in epoxy is created at a 1/40th scale. This mock-up gets all ship complex parts, including propellers in noble metal. On this hull are placed 6 platinum anodes, which are linked to independent dc generators (providing currents up to $50 \mathrm{~mA}$ ). Thirty-six polarizable steel cathodes are also set on the hull to simulate paint defects (positions chosen from statistical study of real cases). They can individually be activated thanks to separated connectivity wires. This can simulate a general defect reaching $7 \%$ of the hull total surface (Fig. 7).

Unfortunately, those wires make different coils appearing around the mock-up, which fully overlay the CRM. Only experimental electric field comparisons at this deeper depth can be done. This phenomenon does not happen for real vessels because cathodic currents circulate into the hull (and not in wires), avoiding this "coil effect".

The hull is partially immerged in salted and bubbled water ( $\left.\sigma=0.13 \mathrm{~S} . \mathrm{m}^{-1} ; \mu=\mu_{0}\right)$ and electromagnetic measurements are made below the hull. This has been realized at the Naval Research Laboratory (Key West) of the US Navy, with a partnership with the French Armament Delegation (Fig. 8).

From the closest electric field measurement, corrosion diagnosis leads to good results on the mock-up, with less than 5\% error on hull's current densities. Those results are available in a previous publication [1]. From these boundary conditions the magnetic induction is computed on the deeper line (Fig. 9).

As said before, experimental validations and FEM do not give usable results. Luckily, we have some points of comparison: analytical results (simplifying the structure to a dipole) give same

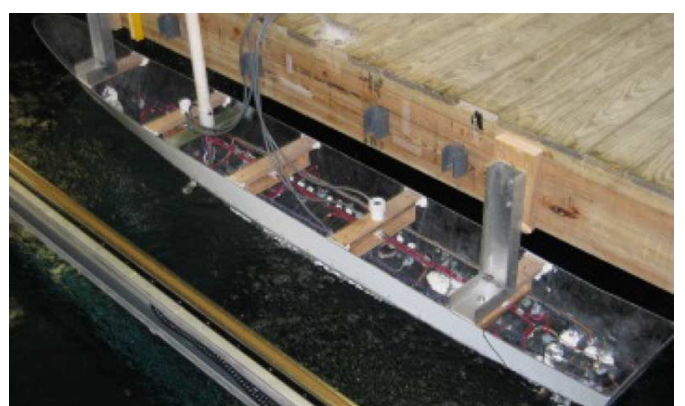

Fig. 8. Mock-up in measurement conditions.

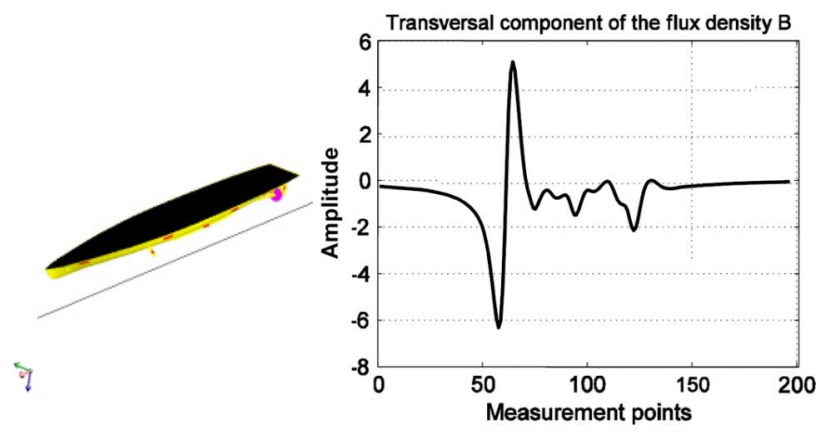

Fig. 9. Computation line below the hull (left) and transversal magnetic induction calculation (tesla) (right).

amplitudes. Then, the magnetic measurements on a real vessel give similar curve shapes. Finally, electric field computation on the same line fits results obtained with other checked modeling tools (based on BEM). A modeling of a real hull with magnetic measurements would be a good conclusion for this study.

\section{CONCLUSION}

This paper has presented a powerful tool to get magnetic induction in conductors, with great advantages in specific conditions. Such examples are computation in sources or in external problems. This is the case for underwater structures under cathodic protection. Some improvements could be made on computational aspects: tangential electric field evaluation could be more precise with a better treatment of the neighborhood meshing (with second order integrations). This method could be applied to other scientific domains, which contain conductive medias, such as neurobiology for example.

\section{REFERENCES}

[1] A. Guibert, J.-L. Coulomb, O. Chadebec, C. Rannou, and R. P. Nogueira, "Ships hull corrosion diagnosis from close measurements of electric potential in the water," IEEE Trans. Magn., vol. 45, no. 3, pp. 1828-1831, Mar. 2009.

[2] B. Haghi-Ashtiani, "Méthodes d'assemblage rapide et de résolution itérative pour un solveur adaptatif en équations intégrales de frontiéres destiné à l'électromagnétisme," Ph.D. dissertation, Ecole Centrale de Lyon, 1998.

[3] O. Chubar, P. Elleaume, and J. Chavanne, "A three-dimensionnal magnetostatics computer code for insertion devices," J. Synchrotron Rad., vol. 5, pp. 481-484, 1998.

[4] J. Creus, R. Sabot, and P. Refait, "Corrosion et protection des métaux en milieu marin," Techniques De L'ingénieur, Corrosion Et Vieillissement, vol. COR1, no. COR620, pp. COR620.1-COR620.14, 2004.

[5] N. G. Zamani, J. M. Chuang, and J. F. Porter, "BEM simulation of cathodic protection systems employed in infinite electrolytes," Int. J. Numer. Methods Eng., vol. 24, no. 3, pp. 605-620, Mar. 1987. 